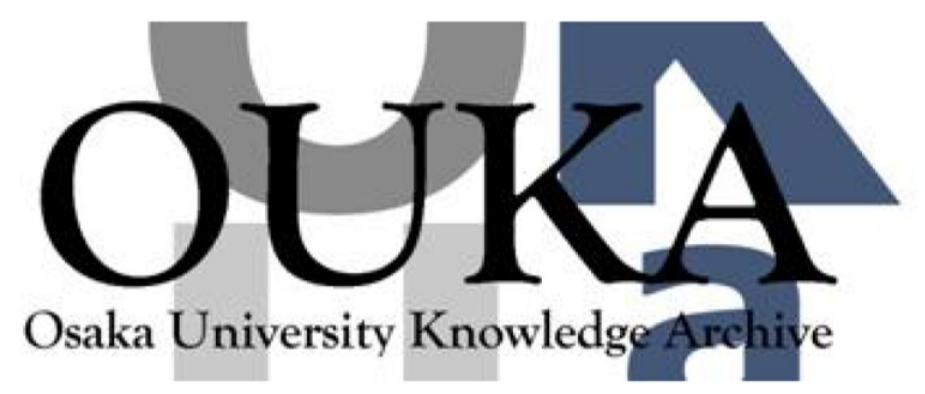

\begin{tabular}{|c|l|}
\hline Title & $\begin{array}{l}\text { Flexible mirrorless laser based on a free- } \\
\text { standing film of photopolymerized cholesteric } \\
\text { liquid crystal }\end{array}$ \\
\hline Author(s) & $\begin{array}{l}\text { Matsui, Tatsunosuke; Ozaki, Ryotaro; Funamoto, } \\
\text { Kazuhiro et al. }\end{array}$ \\
\hline Citation & Applied Physics Letters. 81(20) p.3741-p. 3743 \\
\hline Issue Date & $2002-11-04$ \\
\hline oaire:version & VoR \\
\hline URL & https://hdl.handle.net/11094/75832 \\
\hline rights & \\
\hline Note & \\
\hline
\end{tabular}

Osaka University Knowledge Archive : OUKA

https://ir. Library. osaka-u. ac. jp/

Osaka University 


\section{Flexible mirrorless laser based on a free-standing film of photopolymerized cholesteric liquid crystal}

Cite as: Appl. Phys. Lett. 81, 3741 (2002); https://doi.org/10.1063/1.1522498

Submitted: 08 July 2002 . Accepted: 27 September 2002 . Published Online: 04 November 2002

Tatsunosuke Matsui, Ryotaro Ozaki, Kazuhiro Funamoto, Masanori Ozaki, and Katsumi Yoshino

\section{ARTICLES YOU MAY BE INTERESTED IN}

Cholesteric liquid crystal laser with wide tuning capability

Applied Physics Letters 86, 161120 (2005); https://doi.org/10.1063/1.1897439

Cholesteric liquid crystal/polymer dispersion for haze-free light shutters Applied Physics Letters 60, 3102 (1992); https://doi.org/10.1063/1.106765

Cholesteric liquid-crystal laser as an optic fiber-based temperature sensor Applied Physics Letters 85, 2691 (2004); https://doi.org/10.1063/1.1781363

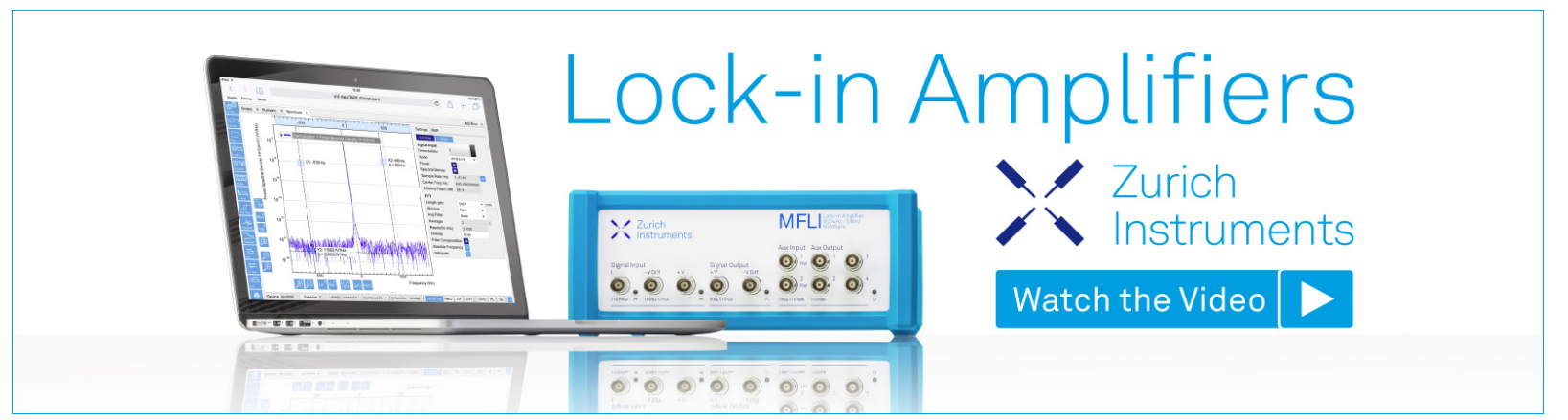




\title{
Flexible mirrorless laser based on a free-standing film of photopolymerized cholesteric liquid crystal
}

\author{
Tatsunosuke Matsui, Ryotaro Ozaki, Kazuhiro Funamoto, Masanori Ozaki, ${ }^{\text {a) }}$ \\ and Katsumi Yoshino \\ Department of Electronic Engineering, Graduate School of Engineering, Osaka University, \\ 2-1 Yamada-Oka, Suita, Osaka 565-0871, Japan
}

(Received 8 July 2002; accepted 27 September 2002)

\begin{abstract}
Optically pumped mirrorless laser action has been observed in a dye-doped flexible free-standing film of photopolymerized cholesteric liquid crystal (PCLC). In the PCLC film, self-organized helical structure acts as one-dimensional (1D) photonic crystal. At high excitation intensity above the threshold, a laser action is observed at an edge of the 1D photonic band of the PCLC helical structure. This PCLC film laser possesses an excellent mechanical flexibility, and the laser action is also observed in a bent film of PCLC. This implies that the one-dimensional periodic structure for the laser action is maintained even in the deformed film. Using such flexibility of the PCLC film, a focusing effect of laser emission is demonstrated in a circularly deformed film. Moreover, the helical pitch of the PCLC has no temperature dependence in contrast to that of unpolymerized cholesteric liquid crystal. This means that the operation wavelength of laser action is thermally stable, which is the great advantage for the device application. (C) 2002 American Institute of Physics.
\end{abstract}

[DOI: $10.1063 / 1.1522498]$

Since proposals of Yablonovitch ${ }^{1}$ and John, ${ }^{2}$ a photonic crystal (PC) which is made from a periodic dielectric structure with a periodicity in a range of optical wavelength has attracted much attention from both fundamental and practical points of view. ${ }^{3}$ In the PC, the propagation of light is inhibited under the Bragg condition, which results in an appearance of an optical stop band or a photonic band gap (PBG). In the PBG, spontaneous emission is inhibited, so that a lowthreshold laser action can be expected. ${ }^{1,4}$

Cholesteric liquid crystal (CLC) and chiral smectic liquid crystals such as ferroelectric liquid crystal, which have a periodic helical structure with a periodicity of the optical wavelength, can be regarded as a one-dimensional (1D) PCs. If liquid crystal has a chirality in the molecular structure, they form a 1D periodic helical structure spontaneously. In the CLC, liquid crystal molecules align their molecular long axes (director) homogeneously in the plane perpendicular to the helical axis and rotate the direction of director continuously along the helical axis. In such liquid crystals with the helical structure, a circularly polarized light with the same handedness as the helix propagating along a helical axis is selectively reflected (selective reflection) and a stop band appears. The wavelength $\lambda$ of the stop band is by $\lambda=n p$, where $n$ and $p$ are an averaged refractive index of liquid crystal and the pitch of helix, respectively, and $n$ $=\sqrt{\left(n_{o}^{2}+n_{e}^{2}\right) / 2}$, where $n_{o}$ and $n_{e}$ are ordinary and extraordinary refractive indices of liquid crystal, respectively.

The PC having a 1D periodic structure is called a 1D PC. Although, in the 1D PC, the complete localization of photon such as in the three-dimensional PC cannot be achieved, the laser action can be expected at the edge of stop band, where the photon group velocity approaches zero. ${ }^{5}$ Recently, the

${ }^{a)}$ Electronic mail: ozaki@ele.eng.osaka-u.ac.jp laser actions have been observed in both $\mathrm{CLC}^{6-8}$ and chiral smectic phases. ${ }^{9}$ In such materials, a $1 \mathrm{D}$ periodic helical structures act as laser cavities and distributed feedback laser action was achieved at the photonic stop band edges. In this study, the laser emission of a flexible free-standing film of photopolymerized CLC (PCLC) is studied.

For the fabrication of PCLC, two types of photopolymerizable CLC mixtures (Merck KGaA) were used, which have right-handed helix and reflection bands around 779 and 440 $\mathrm{nm}$. The helical pitch of the CLC sample, that is, the wavelength of the optical stop band can be adjusted by mixing these two CLC compounds at a proper ratio. As a laser dye dopant in the CLC, [2-[2-4-(Dimethylamino)phenyl] ethenyl]-6-methyl-4H-pyran-4-ylidene propanedinitrile (DCM) (Exciton) was used. The concentration of the dye was 0.4 wt. \%. The free-standing CLC polymer film was prepared as follows. The monomer sample was inserted by a capillary action into the sandwiched cell that is composed of two grass plates. In order to obtain a homogeneously aligned cell, the surfaces were coated with a polyimide (Japan Synthetic Rubber, AL1254) and rubbed. The cell gap was 25 $\mu \mathrm{m}$. The CLCs in this cell align their director parallel to the glass plates, that is, the helical axis is perpendicular to the glass substrates. UV light irradiation was performed using a Xe lamp to induce the photopolymerization of the UVcurable CLC monomer. After UV light irradiation, two glass substrates were removed and the free-standing PCLC film was obtained.

Figure 1(a) shows the transmission spectrum of the dyedoped PCLC. The drop of the transmittance due to the selective reflection is observed around $600 \mathrm{~nm}$, which corresponds to the longer wavelength edge of the stop band. Transmittance in shorter wavelength than about $570 \mathrm{~nm}$ is suppressed by the absorption of the doped laser dye. Figure 1(b) shows the emission spectra of the dye-doped PCLC film 


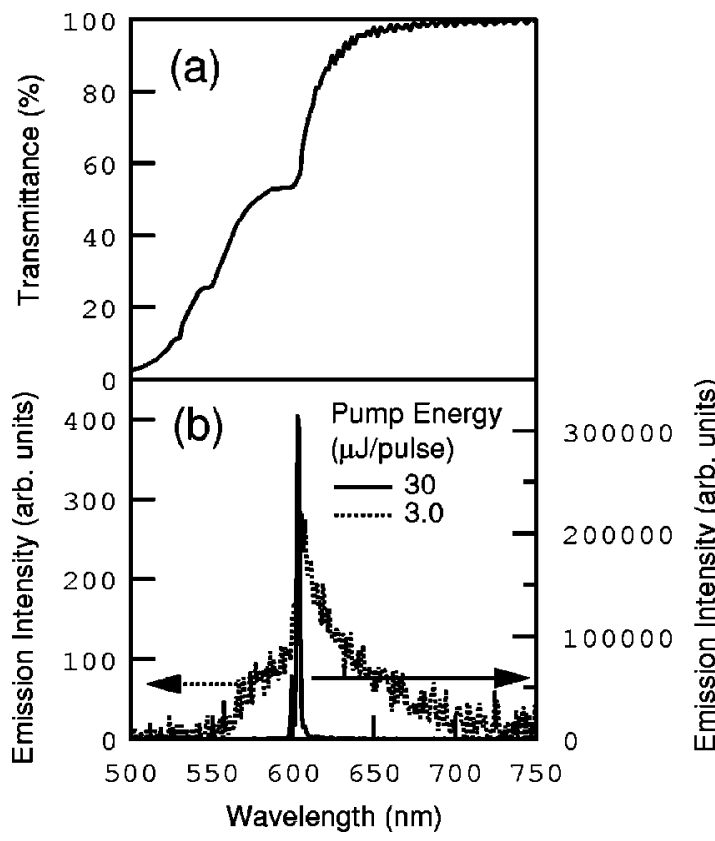

FIG. 1. (a) Transmission spectrum of the dye-doped PCLC. (b) Emission spectra of the dye-doped PCLC film at below $(3.0 \mu \mathrm{J} /$ pulse, dotted line) and above (30 $\mu \mathrm{J} /$ pulse, solid line) the threshold pump pulse intensity.

for various excitation pulse intensities. A second harmonic light of $Q$-switched Nd:yttritium-aluminum-garnet laser (Spectra Physics, Quanta-Ray INDI) was used for an excitation, whose wavelength, pulse width and pulse repetition frequency were $532 \mathrm{~nm}, 8 \mathrm{~ns}$, and $10 \mathrm{~Hz}$, respectively. The illumination area on the sample was about $0.2 \mathrm{~mm}^{2}$. The excitation laser beam irradiated the sample at an angle of about $45^{\circ}$ with respect to the cell plate normal. The emission spectra from the dye-doped PCLC film were measured from an opposite side of the cell using a charge coupled device multichannel photodetector (Hamamatsu Photonics, PMA11) having a spectral resolution of $3 \mathrm{~nm}$. The collecting direction was perpendicular to the cell surface, which is along the helical axis of PCLC. For low excitation intensity $(<3.0$ $\mu \mathrm{J} /$ pulse), the spectrum is dominated by a broad spontaneous emission (SE). The suppression of SE due to the stop band is observed in shorter wavelength than $600 \mathrm{~nm}$, which coincides with the result of the transmission spectrum shown in Fig. 1(a). At high excitation intensity (30 $\mu \mathrm{J} /$ pulse), laser action is observed at the edge of the dip as shown in solid line. The full width at half maximum of the emission peak is about $2 \mathrm{~nm}$, which is limited by the spectral resolution of our experimental setup. At lower excitation intensities, the emission intensity increases in proportion to the pump energy. Above the threshold at a pump pulse intensity of about $3 \mu \mathrm{J} /$ pulse, the emission intensity increases nonlinearly. The linewidth of the emission spectrum also drastically decreases above the threshold. These results confirm that laser action occurs above the threshold of the pump intensity at the edge of the photonic stop band overlapping with the spontaneous emission spectrum.

Figure 2 shows a photograph of the laser emission from the free-standing dye-doped PCLC film at above the threshold pump energy. It should be noted that mirrorless laser action is achieved without any substrates, and the operation of this laser needs no optical setup for such as fixation, align-

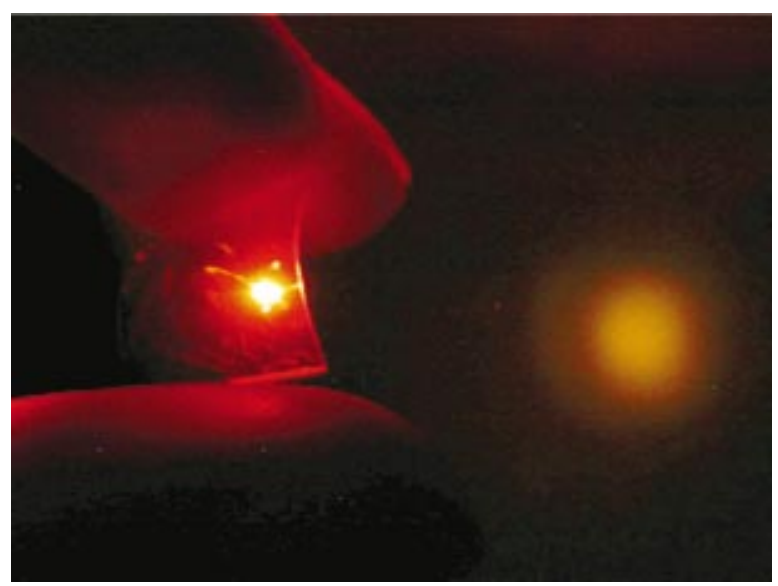

FIG. 2. (Color) The photograph of laser action from the free-standing film of PCLC at above threshold pump pulse energy. The far-field pattern of laser emission can also be observed. The screen on which the spot of laser emission is projected is located at $8 \mathrm{~cm}$ distance from the film.

ment, temperature control, and so on. It should also be noted that the laser emission is observed even when PCLC film is bent. This suggests that $1 \mathrm{D}$ helical structure necessary for the laser action is maintained even in the deformed film. The influences of the mechanical bending of the film on the laser action will be presented elsewhere. This flexibility may enable us to fabricate optical devices with functionalities.

In this study, using such a flexible characteristic of PCLC film, the focusing effect of laser emission in a circularly deformed PCLC film was demonstrated. In 1D PC, only the component of the light propagating along the periodic structure satisfies the Bragg condition, so that laser emission with small spatial divergence can be observed. ${ }^{10}$ This implies that if a flat PCLC film is excited with a stripe-shaped laser beam, the profile of the laser emission from the PCLC film is also stripelike. On the contrary, if a circularly curved PCLC film is irradiated by a stripelike excitation beam, the laser emission from each points on the film will be focused at the focal point of the curvature. These effects were confirmed experimentally as follows.

A collimated laser beam for an excitation of a laser dye passed through a rectangular-shaped slit in order to form a stripelike excitation beam. The intensity of the pump laser beam was spatially homogeneous in the excitation beam area so that the pump energy is equal throughout the pumped area. Figures 3(a) and 3(b) show the photographs of laser action of flat and circularly bent PCLC film excited by stripelike excitation beam, respectively. The radius $R$ of the curvature of the PCLC film in Fig. 3(b) was $15 \mathrm{~mm}$. The illumination area on the sample was about (a) $2 \times 0.5 \mathrm{~mm}^{2}$ and (b) $3 \times 0.5 \mathrm{~mm}^{2}$, respectively. The screen on which the spot of laser emission was projected was located at (a) 20 and (b) $15 \mathrm{~mm}$ (focal point of the curvature) distance from the film, respectively. In the case of flat PCLC film, a stripelike far-field pattern of the laser emission was observed. On the other hand, in the case of circularly bent PCLC film, a round spot of the laser emission was observed at a focal point of the film curvature of the PCLC. These results imply that the laser emission from each point in the excited area on the film was focused on a point and the focusing of laser action was realized. The focal length of this focusing PCLC laser can be controlled arbitrary by changing a radius of the 

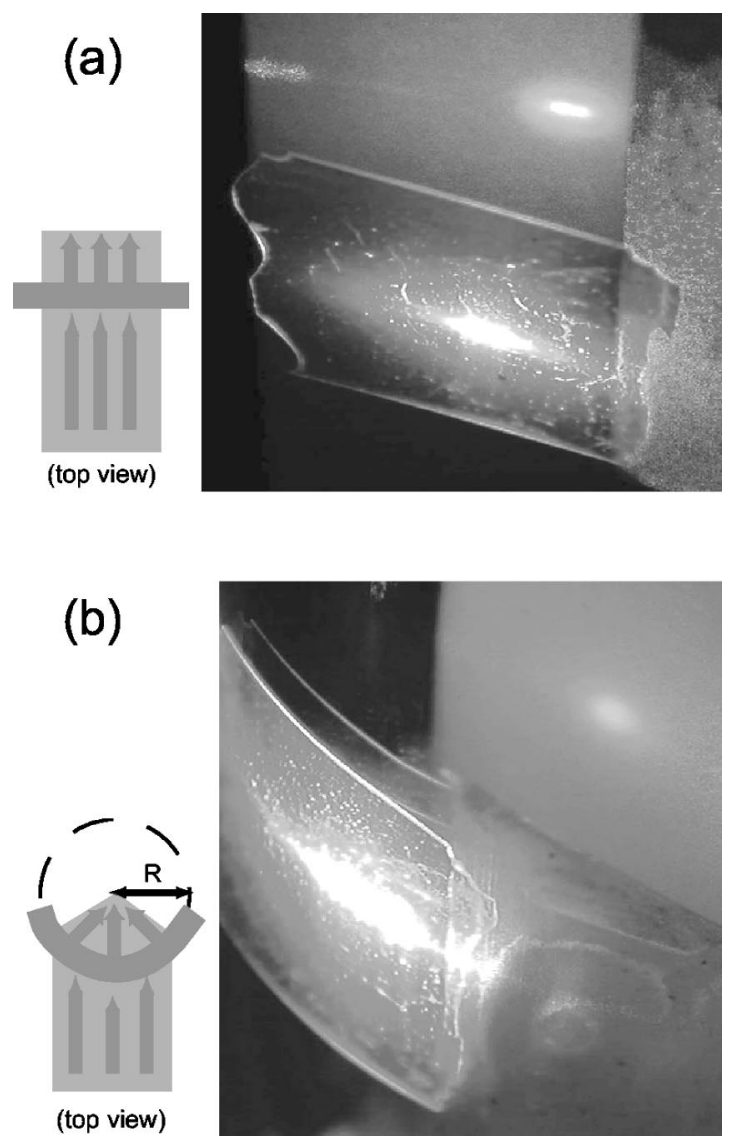

FIG. 3. The photograph of laser action from the (a) flat and (b) circularly deformed free-standing film of PCLC at above threshold pump pulse energy. The far-field (a) stripelike shaped and (b) round spot pattern of the laser emission were also observed. Schematic representations of laser action from top view are also shown.

curvature. Moreover, this focusing effect of circularly deformed PCLC film laser can be applied for various another configurations. For example, if the PCLC is formed into spherical shape, parabolic focusing laser should be realized. This flexibility of PCLC laser may enable us to fabricate optical devices.

From the practical point of view, the thermal stability is important for the laser action. Figure 4 shows the temperature dependence of the emission peak wavelength of PCLC (closed symbols) and unpolymerized CLC mixture (open symbols). The unpolymerized CLC mixture sample was composed by nematic liquid crystal mixture E44 (Merck) as a liquid crystal host, S811 (Merck) as a chiral dopant and DCM as a laser dye. It should be noted that the shift of the emission peak wavelength is negligible $\left(0.09 \mathrm{~nm} /{ }^{\circ} \mathrm{C}\right)$ over the temperature range of about $40^{\circ} \mathrm{C}$ in the case of PCLC film. On the other hand, in the unpolymerized CLC sample, the wavelength of stop band edge shifted to shorter wavelength $\left(15.7 \mathrm{~nm} /{ }^{\circ} \mathrm{C}\right)$ due to the shrinkage of helical pitch, and laser action was not observed at higher than $28^{\circ} \mathrm{C}$. This thermal stability of the lasing wavelength in the PCLC originates from fixing of the helical structure by closslinking liquid

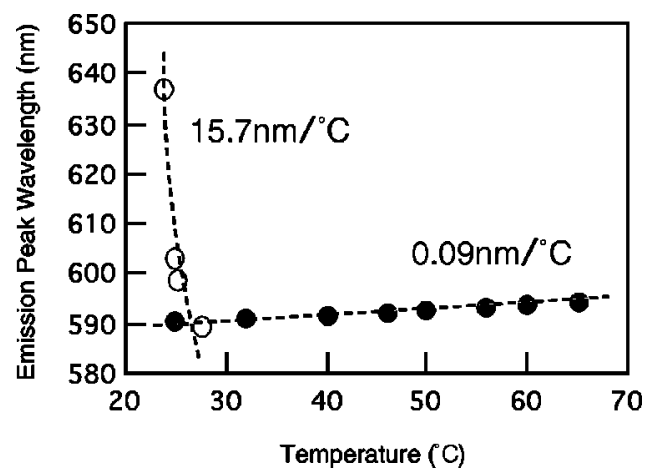

FIG. 4. Temperature dependence of the lasing emission peak wavelength of the PCLC film (closed symbols) and unpolymerized CLC mixture (open symbols).

crystal molecules each other, which is favorable from the view point of the laser device application.

In conclusion, optically pumped mirrorless laser action has been observed in a dye-doped flexible free-standing film of PCLC. In the PCLC film, self-organized helical structure acts as 1D PC. At high excitation intensity above the threshold, a laser action is observed at an edge of the 1D photonic band of the PCLC helical structure. This PCLC film laser possesses an excellent mechanical flexibility, and the laser action is also observed in a bent film of PCLC. This implies that 1D periodic structure for the laser action is maintained even in the deformed film. Using such flexibility of the PCLC film, a focusing effect of laser emission is demonstrated in a circularly deformed film. Moreover, the helical pitch of the PCLC has no temperature dependence in contrast to that of unpolymerized cholesteric liquid crystal. This means that the operation wavelength of laser action is thermally stable, which is the great advantage for the device application.

This work is supported by a Grant-in-Aid for Scientific Research from the Japan Ministry of Education, Culture, Sports, Science and Technology (14350165). The authors would like acknowledge Merck KGaA for providing the photopolymerizable CLC materials.

${ }^{1}$ E. Yablonovitch, Phys. Rev. Lett. 58, 2059 (1987).

${ }^{2}$ S. John, Phys. Rev. Lett. 58, 2486 (1987).

${ }^{3}$ J. D. Joannopoulos, R. D. Meade, and J. N. Winn, Photonic Crystals: Molding the Flow of Light (Princeton University Press, Princeton, NJ, 1995).

${ }^{4}$ J. Martorell and N. M. Lawandy, Phys. Rev. Lett. 65, 1877 (1990).

${ }^{5}$ J. P. Dowling, M. Scalora, M. J. Bloemer, and C. M. Bowden, J. Appl. Phys. 75, 1896 (1994).

${ }^{6}$ V. I. Kopp, B. Fan, H. K. Vithana, and A. Z. Genack, Opt. Lett. 23, 1707 (1998).

${ }^{7}$ B. Taheri, A. F. Munoz, P. Palffy-Muhoray, and R. Twieg, Mol. Cryst. Liq. Cryst. 358, 73 (2001).

${ }^{8}$ H. Finkelmann, S. T. Kim, A. Munoz, P. Palffy-Muhoray, and B. Taheri, Adv. Mater. 13, 1069 (2001).

${ }^{9}$ M. Ozaki, M. Kasano, D. Ganzke, W. Haase, and K. Yoshino, Adv. Mater. 14, 306 (2002).

${ }^{10}$ V. I. Kopp, A. Z. Genack, and Z.-Q. Zhang, Phys. Rev. Lett. 86, 1753 (2001). 